\title{
Primary pulmonary epithelioid malignant peripheral nerve sheath tumor in a child: a case report
}

\author{
Moyan Zhang ${ }^{1 \#}$, Peng Song ${ }^{1 \#}$, Lin Yang ${ }^{2}$, Wei Guo ${ }^{1}$, Ying $\mathrm{Ji}^{1}$, Huayu $\mathrm{He}^{1}$, Shugeng Gao ${ }^{1}$ \\ ${ }^{1}$ Department of Thoracic Surgery, ${ }^{2}$ Department of Pathology, National Cancer Center/National Clinical Research Center for Cancer/Cancer \\ Hospital, Chinese Academy of Medical Sciences and Peking Union Medical College, Beijing 100021, China \\ \#These authors contributed equally to this work. \\ Correspondence to: Shugeng Gao. Department of Thoracic Surgery, National Cancer Center/National Clinical Research Center for Cancer/Cancer \\ Hospital, Chinese Academy of Medical Sciences and Peking Union Medical College, Beijing 100021, China. Email: shugenggao@126.com.
}

\begin{abstract}
Primary pulmonary malignant peripheral nerve sheath tumors (MPNSTs) are uncommon sarcomas originating from intrapulmonary nerve sheath and have been rarely observed in children. Here, we report a case of a 16-year-old child who presented with a mass located in the upper lobe of right lung. The patient underwent a sleeve lobectomy and the pathological diagnosis of the tumor was primary pulmonary epithelioid MPNST. Despite the radical resection, multiple suspected metastasis occurred in heart, lung and muscles less than 2 years after the operation and the patient died 6 months after the metastasis.
\end{abstract}

Keywords: Malignant peripheral nerve sheath tumor (MPNST); lung; child; surgery

Submitted Sep 06, 2019. Accepted for publication Jan 14, 2020.

doi: 10.21037/atm.2020.02.56

View this article at: http://dx.doi.org/10.21037/atm.2020.02.56

\section{Introduction}

Malignant peripheral nerve sheath tumor (MPNST) is a soft-tissue sarcoma arising from the peripheral nerve sheath, accounting for approximately $5 \%$ to $10 \%$ of all sarcomas. MPNSTs can occur at any age, mostly at the age of 20 and 50 years old (1). There is no difference in the incidence between male and female and approximately half of all cases of MPNST occur in patients with neurofibromatosis-1 (NF-1) (1). Primary pulmonary MPNSTs are extremely rare and seldom occurred in a child (2). In this paper, we report a case of primary pulmonary MPNST in a 16 -year-old child who was treated with radical surgery and developed multiple metastasis after operation.

\section{Case presentation}

A 16-year-old girl was admitted to our hospital with a 1-year history of cough and 3-month history of intermittent fever. Physical examination of the respiratory system showed clear breath sound on the left lung, decreased breath sound on the right lung with dry rale, slightly biased trachea and dullness to percussion on the right side. There were no signs of pancoast tumor or compression of superior vena cava. There were no clinical signs of neurofibromatosis and no family history of neurofibromatosis. Computed tomography (CT) of the thorax revealed a large mass measuring $4.0 \times 4.8 \mathrm{~cm}$ at the proximal of right upper lobe, which was adjacent to the superior vena cava, trachea and esophagus (Figure 1A,B,C). The bronchoscopic examination showed that the apical segmental bronchus of the right upper lobe was completely obstructed by a mass (Figure 1D). Bronchoscopic biopsy was performed, and the results of histological examination revealed that the tumor was suspected to originate from mesenchymal tissue and the possibility of malignant tumors could not be ruled out.

We performed a right upper lobectomy and end-to-end anastomosis between the right main bronchus and the lower right bronchus under general anesthesia with a single-lumen endotracheal tube. The frozen sections of the surgical margins of the resected trachea and bronchus were negative for malignancy. Gross pathologic examination showed that the tumor, which measured $4.0 \times 4.0 \times 3.0 \mathrm{~cm}$, invaded the segmental bronchus. Microscopic examination revealed 

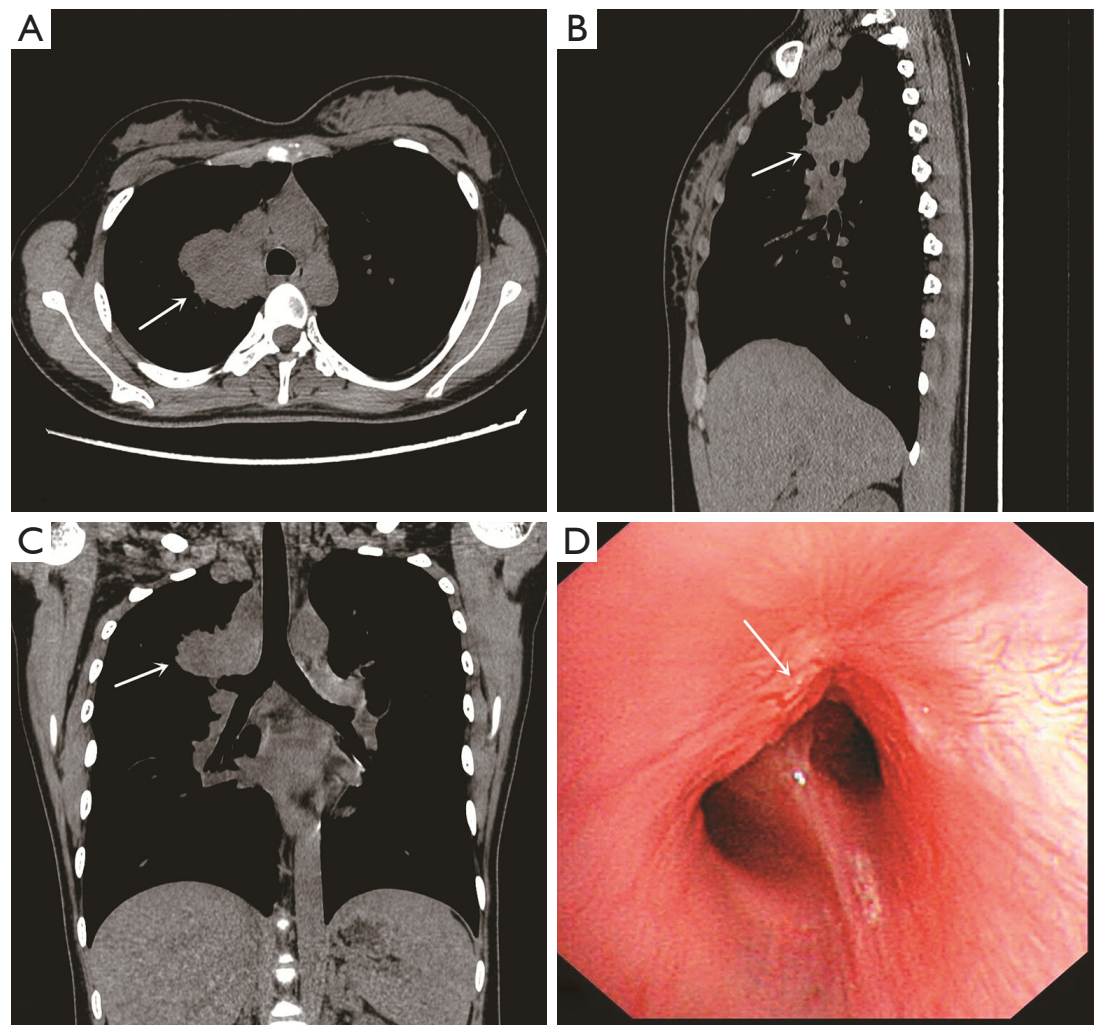

Figure 1 Computed tomography (CT) of chest and bronchoscopy. (A-C) Transverse/sagittal/coronal planes of chest CT revealed a large mass measuring $4.0 \times 4.8 \mathrm{~cm}$ at the proximal of right upper lobe (white arrow); (D) the bronchoscopy revealed that a mass completely obstructed the apical segmental bronchus of the right upper lobe (white arrow).

poorly differentiated epithelioid tumor cells arranged in flakes or nests and dispersed in a fibrillary stroma, with round or oval nucleus, delicate nuclear chromatin and scarce cytoplasm (Figure 2). None of 15 resected lymph nodes were metastatic. Immunohistochemical study showed strong positive for S-100, moderate positive for Vimentin, weak positive for cytokeratin AE/AE3 (CK AE/AE3) and epithelial membrane antigen (EMA), and negative for Calretinin (CR) (Figure 2). The pathological diagnosis was primary pulmonary epithelioid MPNST. The patient underwent an uneventful post-operative course without complications and was followed up every 3-6 months after operation. During the 2-year follow-up, positron emission tomography/computed tomography (PET/CT) revealed multiple lesions with high FDG uptake in heart, lung and muscles less than 2 years after the operation, which were highly suspected to be the metastasis of pulmonary malignant MPNST (Figure 3). Due to refusal to treatment, the lesions were not confirmed to be the metastasis of pulmonary malignant MPNST by pathology and the patient died 6 months later.

\section{Discussion}

MPNSTs can occur in any region of the body, and the most common anatomical sites are the upper and lower extremities, head and neck, and trunk (3). MPNSTs are rare in children, in whom most of the cases are commonly accompanied by NF-1 $(3,4)$. Tumors of nerve sheath originating in the lung account for only $0.2 \%$ of all lung tumor cases (5), most of which are benign lesions. In our case, we reported the occurrence of a MPNST in the lung in a child.

Symptoms of the tumor are usually nonspecific, mainly include dry cough, fever, hemoptysis, dyspnea, and obstructive pneumonia (6). Moreover, some cases may asymptomatic and discovered incidentally on radiographs. The common CT findings of primary pulmonary MPNSTs include a large mass with clear boundary, low-attenuation areas as a result of hemorrhage and necrosis, obstructive 

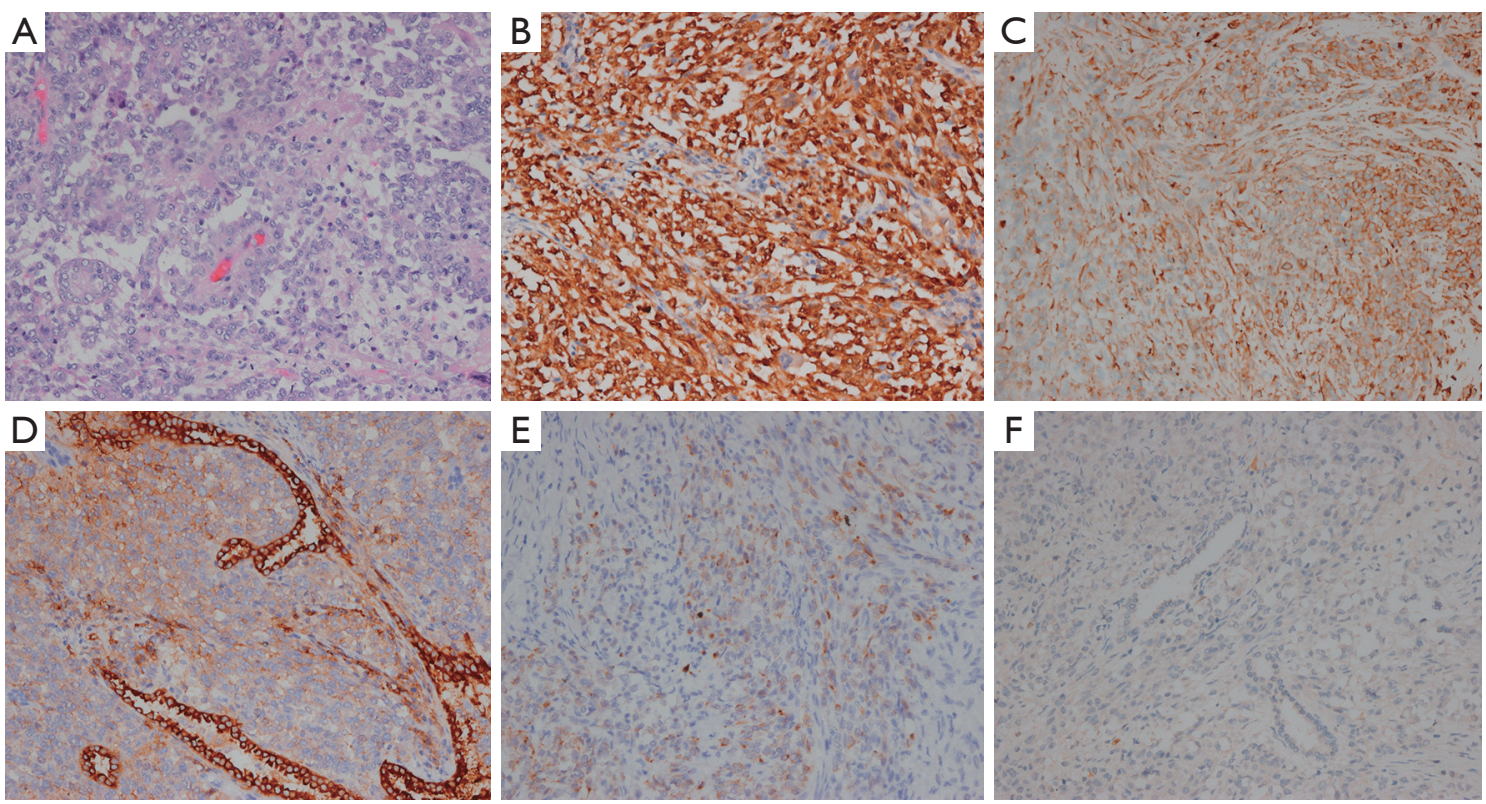

Figure 2 Hematoxylin and eosin staining (HE) and immunohistochemistry (IHC). (A) HE staining showed poorly differentiated epithelioid tumor cells arranged in flakes or nests (×200); (B-F) immunostaining for S-100, Vimentin, EMA, CK(AE1/AE3) and CR, respectively (×200).
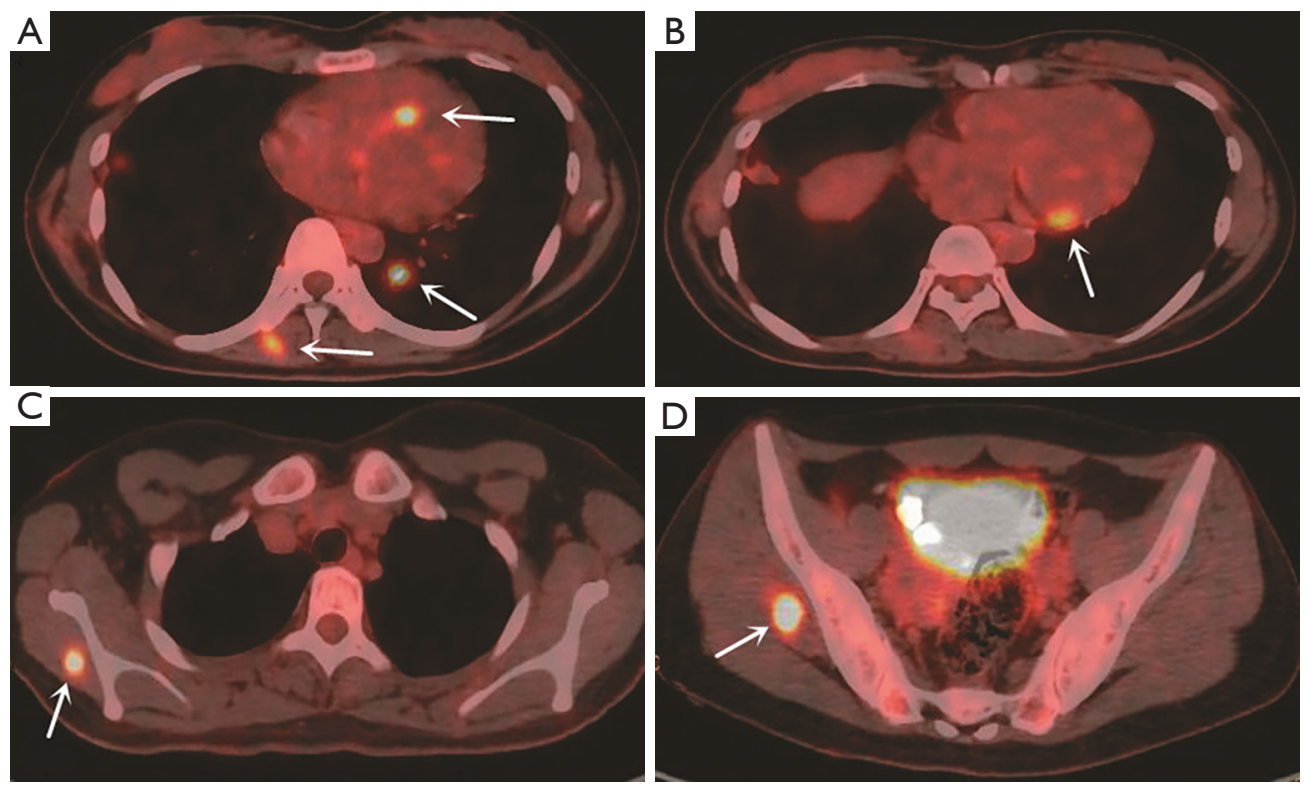

Figure 3 Positron emission tomography/computed tomography (PET/CT) demonstrated multiple lesions with high FDG uptake (SUVmax =12.8), (A-D) lesions with high FDG uptake in heart, lung and muscles (white arrow). FDG, 18-fluorodeoxyglucose.

pneumonia and atelectasis. It is difficult to make a diagnosis by symptoms and radiographic examinations. Pathological examination and immunohistochemistry (IHC) are the gold standard for diagnosis of this tumor.
Microscopic findings of typical cases are composed of spindle cells showing a fascicular growth pattern, often with a branching hemangiopericytoma-like vascular pattern, as well as alternating hypercellular and hypocellular areas (7). 
Diffuse and strong staining for S-100 protein is a typical immunohistochemical feature in MPNST (7). Distinguishingly, our case showed that tumor cells had the characteristics of epithelioid cells and strong positive staining for S-100, based on which it was diagnosed with primary pulmonary epithelioid MPNST.

According to the limited number of reports on primary pulmonary sarcomas, prognosis was poor in primary pulmonary MPNST and 5-year-survival rate was approximately $38 \%$ to $44 \%(6,8-10)$. Its highly invasive nature results in high local recurrence and distant metastasis rate. The primary treatment for MPNST is a complete surgical resection with negative margins, and the surgical margin status is a crucial prognostic factor for MPNST (11). Inci et al. reported that aggressive surgery can significantly improve disease-free survival and two of the cases both achieved long-time disease-free survival (12). Radiotherapy can reduce the local recurrence of MPNSTs and improve the local control rate after operation, whereas the role of chemotherapy alone in MPNSTs has always been controversial. Recently, the use of neoadjuvant chemoradiotherapy followed by surgery has been advocated. In a report by Cuneo et al., the use of preoperative chemoradiotherapy has been shown to be effective and associated with low-risk of metastatic disease and long-term survival (13). The patient in the presented case underwent the radical resection and multiple suspected metastasis of primary pulmonary MPNST were found in heart, lung and muscles less than 2 years after the operation. It is worthy of note that cardiac metastasis of pulmonary MPNST is extremely rare, which, to the best of our knowledge, has never been reported.

\section{Conclusions}

Primary pulmonary MPNSTs rarely occurred in children; yet, the suspicion of MPNST should be raised in a child patient presented with CT findings of a solid mass of lung. Patients with primary pulmonary MPNSTs are lack of specific symptoms and radiological features and the accurate diagnosis depends on histopathological examination and IHC. Due to the high malignant potential, radical surgical resection and close follow-up are required for patients with a primary pulmonary MPNST.

\section{Acknowledgments}

Funding: The study was supported by the grant
2018PT32033 from Institutional Fundamental Research Funds.

\section{Footnote}

Conflicts of Interest: The authors have no conflicts of interest to declare.

Ethical Statement: The authors are accountable for all aspects of the work in ensuring that questions related to the accuracy or integrity of any part of the work are appropriately investigated and resolved. Written informed consent was obtained from the patients for publication of this case report and any accompanying images.

Open Access Statement: This is an Open Access article distributed in accordance with the Creative Commons Attribution-NonCommercial-NoDerivs 4.0 International License (CC BY-NC-ND 4.0), which permits the noncommercial replication and distribution of the article with the strict proviso that no changes or edits are made and the original work is properly cited (including links to both the formal publication through the relevant DOI and the license). See: https://creativecommons.org/licenses/by-nc-nd/4.0/.

\section{References}

1. Bates JE, Peterson CR, Dhakal S, et al. Malignant peripheral nerve sheath tumors (MPNST): a SEER analysis of incidence across the age spectrum and therapeutic interventions in the pediatric population. Pediatr Blood Cancer 2014;61:1955-60.

2. Bartley TD, Arean VM. Intrapulmonary neurogenic tumors. J Thorac Cardiovasc Surg 1965;50:114-23.

3. Enzinger FM, Weiss SW. Malignant tumors of the peripheral nerves. In: Soft tissue tumors. 3rd edition. St Louis: Mosby, 1995:889-9042.

4. Meis JM, Enzinger FM, Martz KL, et al. Malignant peripheral nerve sheath tumors (malignant schwannomas) in children. Am J Surg Pathol 1992;16:694-707.

5. Roviaro G, Montorsi M, Varoli F, et al. Primary pulmonary tumors of neurogenic origin. Thorax 1983;38:942-5.

6. Etienne-Mastroianni B, Falchero L, Chalabreysse L, et al. Primary sarcomas of the lung: a clinicopathologic study of 12 cases. Lung Cancer 2002;38:283-9.

7. Travis WD, Brambilla E, Burke AP, et al. WHO classification of tumours of the lung, pleura, thymus and heart. Fourth edition. IARC Press, 2015. 
8. Janssen JP, Mulder JJ, Wagenaar SS, et al. Primary sarcoma of the lung: A clinical study with long-term follow-up. Ann Thorac Surg 1994;58:1151-5.

9. Guccion JG, Rosen SH. Bronchopulmonary leiomyosarcoma and fibrosarcoma. A study of 32 cases and review of literature. Cancer 1972;30:836-47.

10. Keel SB, Bacha E, Mark EJ, et al. Primary pulmonary sarcoma: A clinicopathologic study of 26 cases. Mod Pathol 1999;12:1124-31.

11. James AW, Shurell E, Singh A, et al. Malignant

Cite this article as: Zhang M, Song P, Yang L, Guo W, Ji Y, He H, Gao S. Primary pulmonary epithelioid malignant peripheral nerve sheath tumor in a child: a case report. Ann Transl Med 2020;8(6):403. doi: 10.21037/atm.2020.02.56 peripheral nerve sheath tumor. Surg Oncol Clin N Am 2016;25:789-802.

12. Inci I, Soltermann A, Schneiter D, et al. Pulmonary malignant peripheral nerve sheath tumour. Eur J Cardiothorac Surg 2014;46:331-2.

13. Cuneo KC, Riedel RF, Dodd LG, et al. Pathologic complete response of a malignant peripheral nerve sheath tumor in the lung treated with neoadjuvant Ifosfamide and radiation therapy. J Clin Oncol 2012;30:e291-3. 